\title{
Abnormal nailfold videocapillaroscopy in patients with antisynthetase syndrome: case reports and review of the literature
}

\author{
Cristina Pomirleanu ${ }^{1,2}$, Georgiana Strugariu', Iulian Resmerita', \\ Andreea Axinte ${ }^{1}$, Codrina Ancuta ${ }^{1,2}$ \\ ${ }^{1}$ Rheumatology 2 Department, Clinical Rehabilitation Hospital, Iasi, Romania \\ 2"Grigore T. Popa" University of Medicine and Pharmacy, Iasi, Romania
}

\begin{abstract}
Classified as a distinct subset of idiopathic inflammatory myopathies, anti-synthetase syndrome is an orphan autoimmune disease characterized by the presence of anti-aminoacyl-tRNA synthetase antibodies associated with a spectrum of clinical features including interstitial lung disease, myositis or dermatomyositis, chronic polyarthritis, fever, mechanic's hands and Raynaud's phenomenon.

The main objective of our study was to evaluate nailfold capillary abnormalities in a series of cases diagnosed with anti-synthetase syndrome, aiming to describe capillaroscopy findings and to identify potential relation with clinical and functional outcomes.

Irrespective of clinical and serological disease subtype, significant capillaroscopic changes may help diagnosis and identify negative prognostic factors, advancing the role of routine capillaroscopy in the decisional algorithm of anti-synthetase syndrome.

Nailfold videocapillaroscopy is able to provide valuable information in patients with anti-tRNA synthetase myositis, suggesting that diffuse microangiopathy represents an early pathobiologic event.
\end{abstract}

Keywords: antisynthetase syndrome, nailfold videocapillaroscopy

\section{INTRODUCTION}

Classified as a distinct subset of idiopathic inflammatory myopathies, anti-synthetase syndrome (AS) is an orphan autoimmune disease characterized by the presence of anti-aminoacyl-tRNA-synthetase antibodies, associated with a spectrum of clinical features including interstitial lung disease, polymyositis (PM) or dermatomyositis (DM), chronic polyarthritis, fever, mechanic's hands and Raynaud's phenomenon (1,2). A complex multidisciplinary approach is typically required for the correct and prompt diagnosis as well as management of this rare condition (2).

Primary damage to microcirculation with subsequent functional and structural abnormalities of both muscle and cutaneous capillaries remains a fundamental event in the pathobiology of either idiopathic DM or AS-related DM $(1,3)$.

Nailfold videocapillaroscopy (NVC) is a non-invasive promising imaging method, currently used for the in vivo assessment of peripheral microcirculation, not only in systemic sclerosis and scleroderma spectrum disorders, but also in other conditions defined by a widespread vasculopathy (4).

Thus, one indication of NVC may be monitoring of treatment and disease activity in DM $(4,5)$. Significant abnormalities, even a typical scleroderma-like pattern, were reported in such patients, including enlarged capillaries, giant loops and ramified capillaries with excessive and extensive neoangiogenesis $(5,6)$. Although the association of capillary anomalies with various signs and symptoms of myositis and visceral involvement was suggested, the role of capillaroscopy in prompting specific relationships between clinical settings and therapeutic responses was validated only for DM, not for PM patients $(6,7)$.

As DM is basically considered a humorally mediated microangiopathy and PM is generally a cytotoxic T-cells mediated muscle-targeted disorder, one 
may assum that capillaroscopy can be useful in differentiating DM from PM $(3,6)$. Indeed, several capillaroscopic studies were performed in patients with inflammatory myopathies, focusing on capillary modifications (6-9); however, typical findings, differences among the entities included in myositis spectrum diseases, as well as their association with a specific myositis subset, clinical and serological patterns are still imprecisely defined. Moreover, only mild capillary abnormalities, eventually comparable to the healthy population were described in PM (6).

The main objective of our study was to evaluate nailfold capillary abnormalities in a series of cases diagnosed with anti-synthetase syndrome.

\section{MATERIAL AND METHODS}

Patients with AS attending our Outpatient Rheumatology Department between September 2015 and March 2016 were enrolled in this prospective study aiming to describe capillaroscopic findings and to identify potential associations with clinical and functional outcomes.

The diagnosis of AS was established if the patient satisfied the following criteria: (a) one determination of anti-tRNA synthetase antibodies in the serum; and (b) presence of interstitial lung disease, myositis or arthritis $(1,2)$. Myositis was classified as polymyositis (PM) or dermatomyositis (DM) according to 1970 Bohan and Peter criteria (3).

All cases were assessed according to the same predefined protocol comprising (i) demographics (age, gender); (ii) AS clinical pattern as well as active manifestations at presentation; (iii) laboratory investigations and imaging (inflammatory and immunologic parameters, serum muscle enzymes, chest X-ray, electromyography); (iv) therapeutic response and functional outcomes.

Nailfold capillaroscopy was systematically performed in each patient by the same trained physician using an Optilia videocapillaroscope equipped with a $\times 200$ optical probe. A full assessment comprised the examination of four fingers (the second through to the fifth) in both hands, following at least 20 minutes at a comfortable room temperature $\left(22-25^{\circ}\right)$; a drop of immersion oil was applied to the nailfold in order to maximize the translucency of the keratin layer; at least two images of the middle of nailfold were captured and analyzed per finger (10).

Capillaroscopic findings were classified as tortuosities (single or multiple crossovers), enlarged loops (irregular or homogeneous increase of capil- lary diameter $\geq 20$ and $<50 \mu \mathrm{m}$ ), giant capillaries (a homogeneously enlarged loop with a diameter $\geq 50$ $\mu \mathrm{m}$ ), microhemorrhages (presence of one or more hemosiderin deposits due to capillary injury or thrombosis), ramified capillaries (branching, bushy, interconnected capillaries, originating from a single capillary), disorganization of the vascular array, capillary loss (reduction of the normal number of capillaries, usually 7-10 per linear millimeter) and avascular area (intercapillary distance $>500 \mu \mathrm{m})(11)$.

All capillary abnormalities were scored using a semiquantitative rating scale, as follows: "0" = no changes; " 1 " = less than $33 \%$ of capillaries presenting alterations/reduction; " 2 " $=33-66 \%$ of capillaries presenting alterations/reduction; " 3 " = more than $66 \%$ of capillaries presenting alterations/reduction; all evaluations were performed per linear millimetre. The disorganization of the vascular array and the avascular areas were scored as present/absent, while the capillary loss was assigned as " 0 " if $\geq 7$ capillaries could be seen per linear millimeter, "1" for 4-6 capillaries/mm, and " 2 " for $\leq 3$ capillaries/mm (11).

Finally, a scleroderma-like pattern, defined as the alteration of the nailfold microvascular network, with enlarged and giant loops, microhemorrhages, capillary loss, neovascularization and architectural disorganization, was recorded as present or absent (11).

\section{RESULTS - CASE REPORTS}

\section{Clinical case 1}

A 23 years-old non-smoking woman diagnosed with Dermatomyositis, an anti-Jo1 and anti-Ro52 positive disease subset, characterized by severe myositis, severe arthritis, Gottron's papules, Raynaud's phenomenon and interstitial lung disease, treated with synthetic DMARDs (Methotrexate, 15 mg once weekly) combined with oral methylprednisolone (16 mg daily). NVC revealed an abnormal pattern with mild loss of capillaries $(7 / \mathrm{mm})$, irregular distribution, frequent dilated loop and several giant loops, ramified capillaries and no hemorrhages (Fig. 1).

\section{Clinical case 2}

A 46-years old nonsmoking woman known with an anti-Jo1, anti-Ro52 seropositive polymyositis characterized by moderate myositis, severe arthritis, Raynaud's phenomenon, interstitial lung disease, receiving Methotrexate (20 mg/week) and oral meth- 


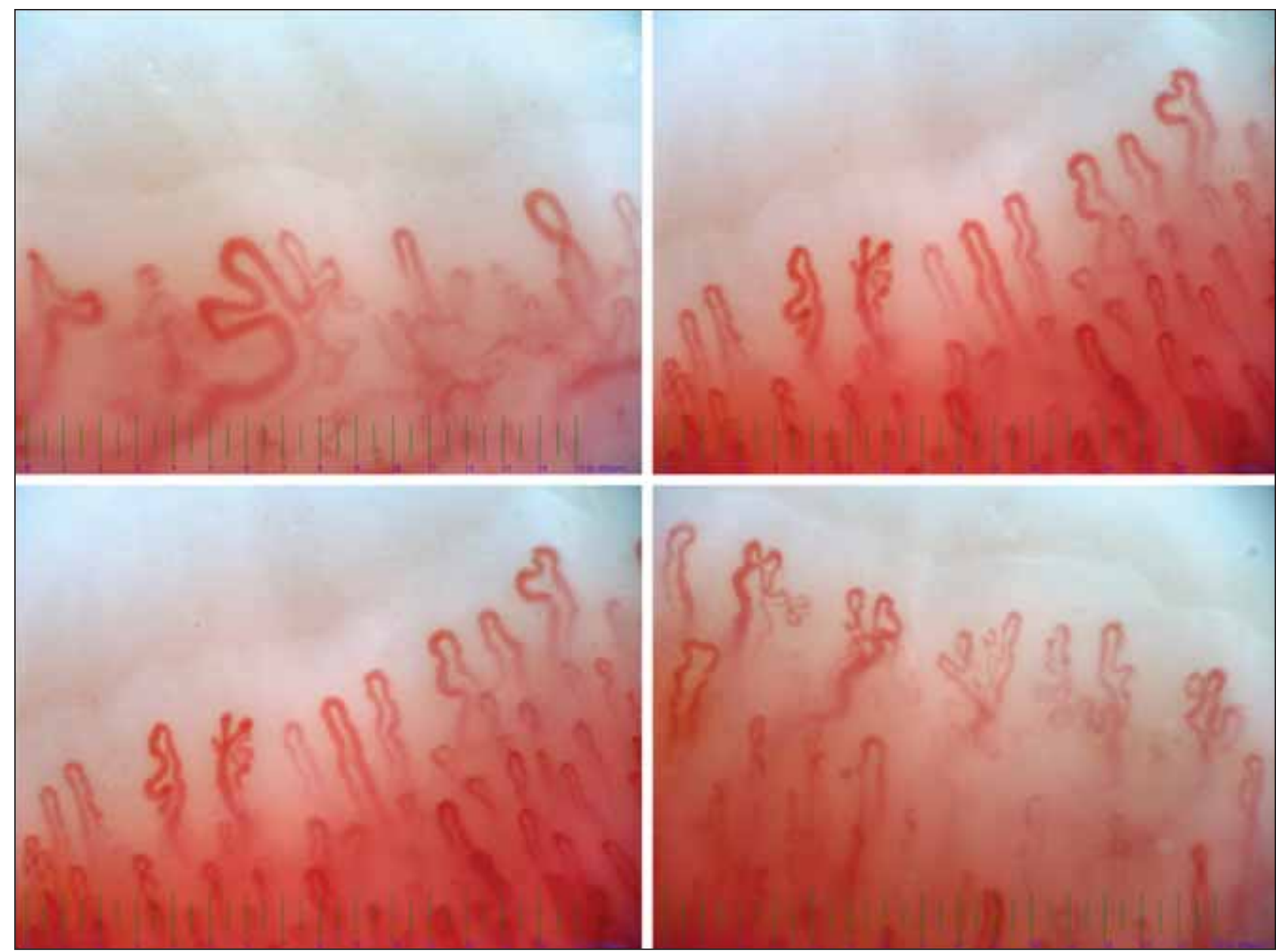

FIGURE 1. Dermatomyositis anti-Jo1 and anti-Ro52 positive with scleroderma-like pattern

ylprednisolone (16 mg/day). NVC revealed normal skin transparency, partially visible subpapillary venous plexus, moderate loss of capillaries $(5 / \mathrm{mm})$, disorganized vessels, frequent giant and ramified capillaries, hemorrhages and hemosiderin deposits (Fig. 2).

\section{Clinical case 3}

A 25-years old smoking woman diagnosed with polymyositis, an anti-PL12 and anti-Ro52 seropositive disease, with mild myositis and arthralgia, receiving oral azathioprine $(100 \mathrm{mg}$ /day) and low dose oral methylprednisolone ( $8 \mathrm{mg}$ /day). NVC revealed normal skin transparency, visible subpapillary venous plexus, irregular distribution and mild loss of capillaries $(7 / \mathrm{mm})$, frequent dilated capillaries, frequent ramified capillaries and no hemorrhages (Fig. 3).

\section{Clinical case 4}

A 39-years old smoking woman, known with an anti-Jo1 and anti-Ro52 seropositive polymyositis characterized by moderate myositis, mild to moder- ate arthritis, Raynaud's, interstitial lung disease. NVC revealed decreased skin transparency, irregular distribution of capillaries, moderate loss of capillaries $(5 / \mathrm{mm})$, giant and ramified capillaries, hemorrhages (Fig. 4).

An abnormal NVC pattern was recorded irrespective of the presence or absence of the Raynaud's phenomenon. The most common abnormalities described in our patients were loss of capillaries, the presence of ramified dilated capillaries and disorganized vessels.

\section{DISCUSSION}

We evaluated three patients with PM and one with DM-related AS and we identified in all cases abnormal videocapillaroscopy. The most frequent capillaroscopic findings in our cases were capillary loss, enlarged and giant capillaries, ramified capillaries and disorganized vascular architecture. We described certain differences between DM and PM patients, with more evident NVC changes in the patient with DM. 


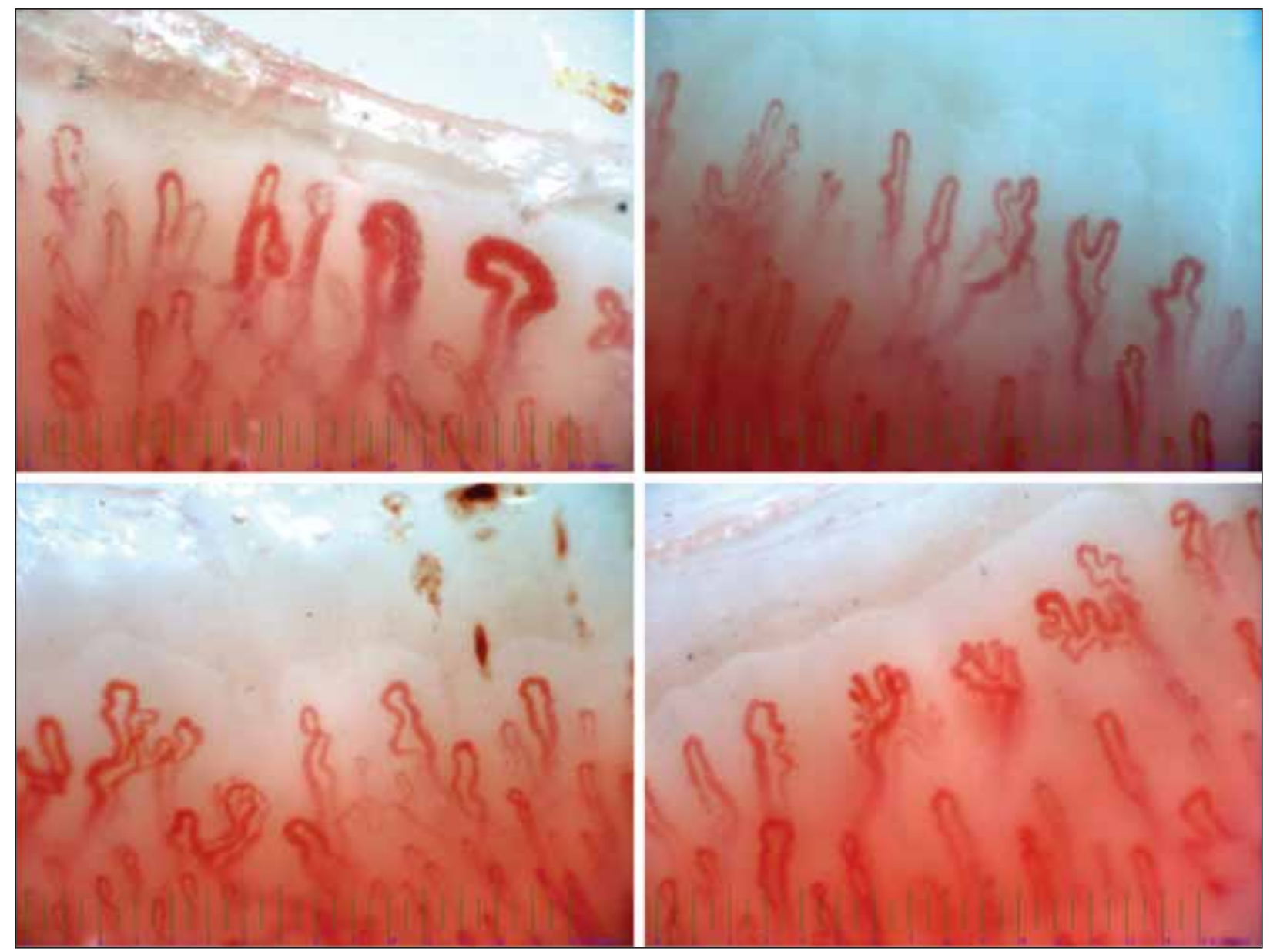

FIGURE 2. Polymyositis anti-Jo1 and anti-Ro52 positive with scleroderma-like pattern

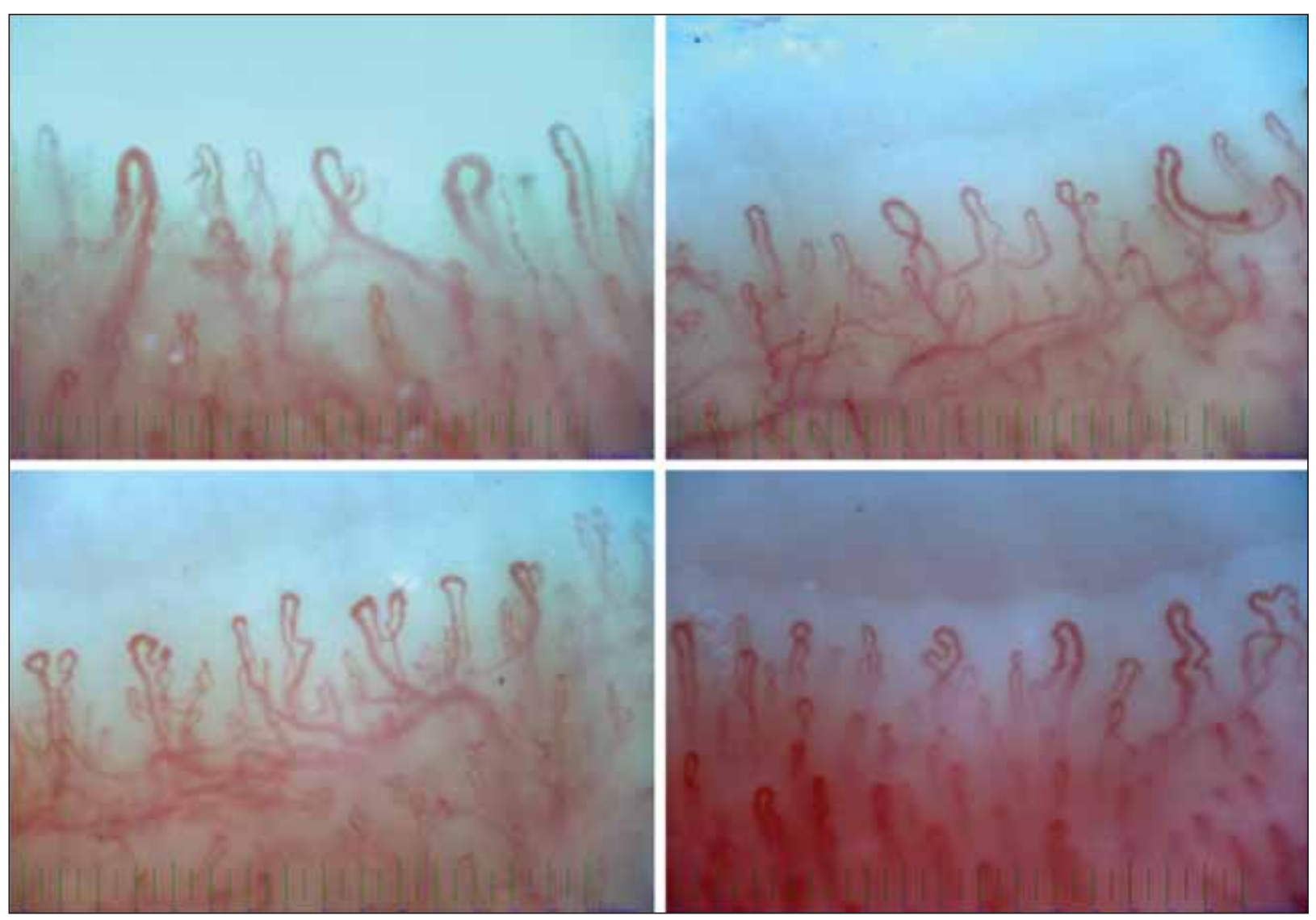




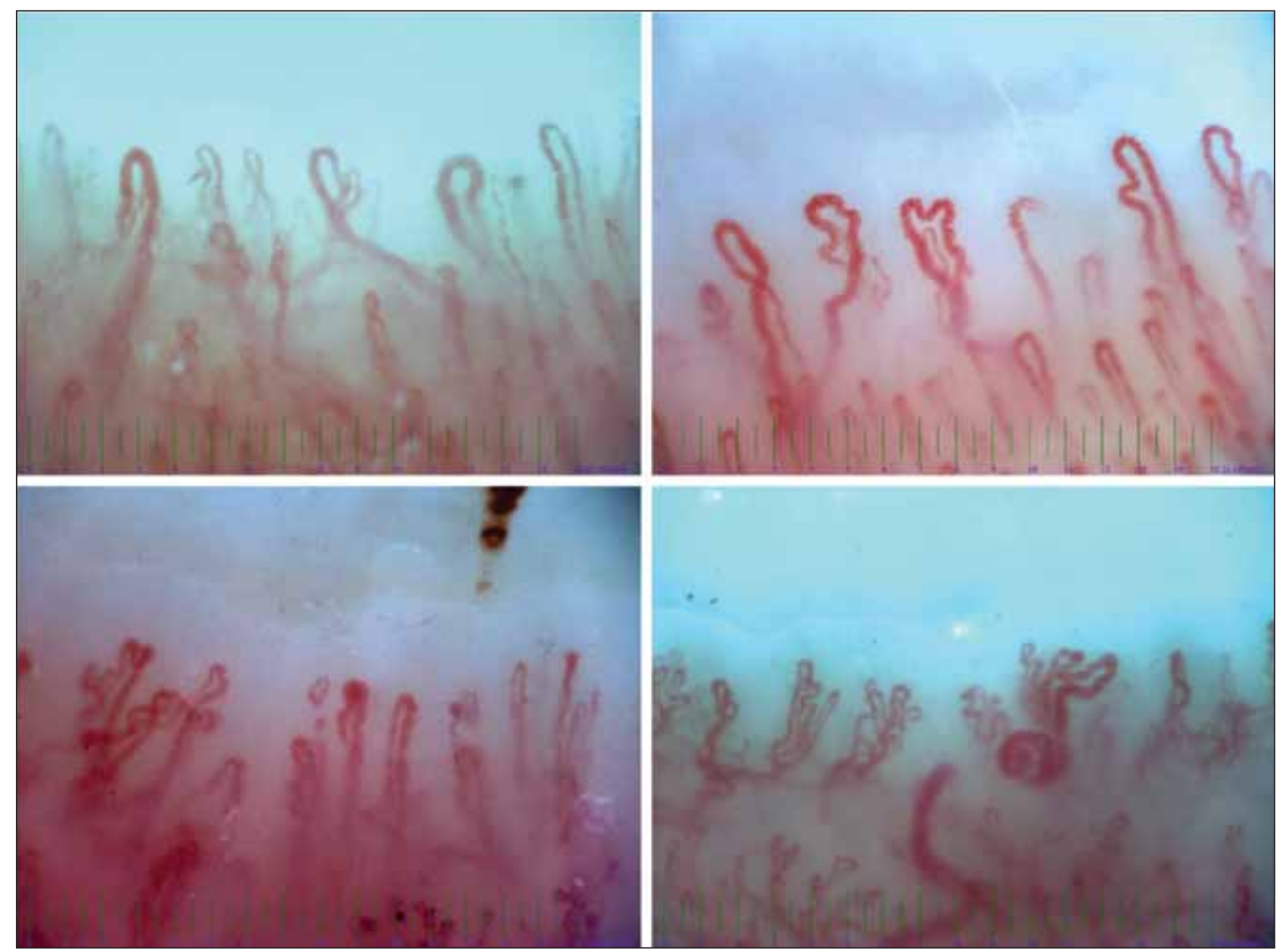

FIGURE 4. Polymyositis anti-Jo1 and anti-Ro52 positive with scleroderma-like pattern

A systematic literature search/review showed several reports about capillaroscopic assessment of patients with myositis.

Thus, capillary changes (graded using a semiquantitative rating scale) were typically seen in patients with inflammatory myopathies (DM, PM, connective tissue diseases overlap), with a higher prevalence of abnormal capillaroscopy features than in healthy controls, particularly higher frequency of ramified capillaries, avascular areas, enlarged capillaries and capillary loss $(6,7)$.

Microangiopathy with or without enlarged capillary loops was identified more frequently in DM than in PM patients (6-8). Furthermore, various capillaroscopic changes were reported in a series of DM, with a predominance of enlarged capillary loops and avascular areas detected with a $\times 20$ magnification stereomicroscope $(5,6,8)$.

Microhemorrhages and capillary enlargement were also dominant in DM, while ramifications and tortuosities were described in both subtypes of myositis $(5,6)$.
A high number of enlarged capillary loops and severe avascular areas correlated with different clinical manifestations, such as interstitial lung disease, Raynaud's phenomenon and arthritis (7,9). Although no relationship was found between capillaroscopic abnormalities and specific autoantibody profile in the majority of studies, a lower capillary density was found in anti-Jo1-positive patients by Mercer et al. (7).

Interestingly, capillary abnormalities such as enlarged capillaries, hemorrhages and capillary loss improved parallel to a decrease in disease activity, while severe changes were usually associated with shorter myositis history; persistence of ramified capillaries was also detected in patients with longstanding disease $(6,7)$.

Finally, another capillaroscopy study described the presence of scleroderma pattern in consecutive DM patients, based on a semiquantitative grading scale (8); the scleroderma pattern was identified in up to $60 \%$ of myositis, related with the presence of Raynaud's phenomenon and interstitial lung disease, especially in juvenile DM (8). 
In addition a significant association between capillaroscopic pattern, high CK levels and severe disease involvement has been reported (12); particularly, capillary loss was significantly associated with muscle and global disease activity, whereas hemorrhages were correlated with skin involvement (12). The presence of branched capillaries is more common in DM, but their presence is not specific and can also be found (only less frequently) in patients with $\operatorname{SSc}(8,12)$.

Certain parameters potentially influence capillaroscopic findings, including the choice of magnification as well as the selection of the nailfold area and number of images per finger $(5,6)$.

Overall, there is a higher prevalence of major capillaroscopic changes (capillary loss, enlarged and giant capillaries and bushy and ramified capillaries) in DM compared to PM and healthy people, probably reproducing the diffuse microangiopathy (5-7).

Returning to our study, we demonstrated abnormal capillaroscopy not only in DM but also in all
PM cases associated with anti-t-RNA synthetase antibodies, suggesting that peripheral vasculopathy may be related to this definite autoantibody profile.

Further studies are required in order to better explain the association of capillaroscopy abnormalities with the myositis subtype (either PM or DM) and with anti-t-RNA synthetase antibody specificity.

\section{CONCLUSIONS}

Nailfold videocapillaroscopy is able to provide valuable information in patients with anti-tRNA synthetase myositis, suggesting that diffuse microangiopathy represents an early pathobiologic event.

Irrespective of clinical and serological disease subtype, significant capillaroscopic changes may improve diagnosis and perhaps identify negative prognostic factors, suggesting a role of routine capillaroscopy in the decisional algorithm of anti-synthetasic syndrome therapy.

Conflict of interest: none declared Financial support: none declared

\section{REFERENCES}

1. Witt L.J., Curran J.J., Strek M.E. The Diagnosis and Treatment of Antisynthetase Syndrome, ClinPulm Med. 2016 Sep; 23(5):218226.

2. Chatterjee S. Antisynthetase syndrome: Not just an inflammatory myopathy. Cleveland Clinic Journal of Medicine 2013; 80(10):655-666.

3. Mahil S., Marks D., McCormack M. et al. Dermatomyositis. Br J Hosp Med (Lond) 2012; 73: C18-22.

4. Chojnowski M.M., Felis-Giemza A., Olesińska M. Capillaroscopy a role in modern rheumatology. Reumatologia. 2016; 54(2): 67-72.

5. De Angelis R. Capillaroscopy in dermatomyositis. In: Cutolo M. Atlas of Capillaroscopy in Rheumatic Diseases. Elsevier, Milan 2010: 115-120.

6. Manfredi A., Sebastiani M., Cassone G. et al. Nailfold capillaroscopic changes in dermatomyositis and polymyositis. Clin Rheumatol 2015; 34:279-284.

7. Mercer L.K., Moore T.L., Chinoy H. et al. Quantitative nailfold video capillaroscopy in patients with idiopathic inflammatory myopathy. Rheumatology 2010; 49:1699-1705.
8. De Angelis R., Cutolo M., Gutierrez M. et al. Different microvascular involvement in dermatomyositis and systemic sclerosis. a preliminary study by a tight videocapillaroscopic assessment. Clin Exp Rheumatol 2012; 30:67-70.

9. Selva-O'Callaghan A., Fonollosa-Pla V., Trallero-Araguas E. et al. Nailfold capillary microscopy in adults with inflammatory myopathy. Semin Arthritis Rheum 2010; 39: 398-404.

10. Smith V., Pizzorni C. The Videocapillaroscopic Technique. In: Cutolo M. Atlas of Capillaroscopy in Rheumatic Diseases. Elsevier, Milan 2010: 25-31.

11. Tavakol M.E., Fatemi A., Karbalaie A. Nailfold Capillaroscopy in Rheumatic Diseases: Which Parameters Should Be Evaluated?

12. Mugii N., Hasegawa M., Matsushita T. et al. Association between nail-fold capillary findings and disease activity in dermatomyositis. Rheumatology 2011; 50:1091-1098. Biomed Res Int 2015; 2015: 974530. 